\title{
Graphene Enclosure of Chemically Fixed Mammalian Cells for Liquid-Phase Electron Microscopy
}

\author{
Patricia Blach ${ }^{1,2}$, Sercan Keskin ${ }^{1}$, Niels de Jonge ${ }^{1,2}$ \\ ${ }^{1}$ INM-Leibniz Institute for New Materials ${ }^{2}$ Department of Physics, Saarland University
}

\section{Corresponding Author}

Niels de Jonge

Niels.deJonge@leibniz-inm.de

\section{Citation}

Blach, P., Keskin, S., de

Jonge, N. Graphene Enclosure

of Chemically Fixed Mammalian

Cells for Liquid-Phase Electron

Microscopy. J. Vis. Exp. (163), e61458,

doi:10.3791/61458 (2020).

\section{Date Published}

September 21, 2020

DOI

$10.3791 / 61458$

\section{URL}

jove.com/video/61458

\section{Abstract}

A protocol is described for investigating the human epidermal growth factor receptor

2 (HER2) in the intact plasma membrane of breast cancer cells using scanning transmission electron microscopy (STEM). Cells of the mammalian breast cancer cell line SKBR3 were grown on silicon microchips with silicon nitride (SiN) windows. Cells were chemically fixed, and HER2 proteins were labeled with quantum dot nanoparticles (QDs), using a two-step biotin-streptavidin binding protocol. The cells were coated with multilayer graphene to maintain a hydrated state, and to protect them from electron beam damage during STEM. To examine the stability of the samples under electron beam irradiation, a dose series experiment was performed. Graphenecoated and non-coated samples were compared. Beam induced damage, in the form of bright artifacts, appeared for some non-coated samples at increased electron dose $D$, while no artifacts appeared on coated samples.

\section{Introduction}

Analysis of membrane protein function is essential for cell biological research, and for drug development. A class of important experiments involves the examination of membrane protein positions in cells. This information can be used to deduce conclusions about the assembly of proteins in protein complexes and their specific locations in the plasma membrane, which, via dynamic assembly and disassembly, drives a wide variety of cellular functions. Among other techniques, light microscopy (LM) and electron microscopy (EM) are used for studying protein functions in cells. LM allows the analysis of whole cells in liquid; however, the resolution is restricted to $200-300 \mathrm{~nm}$ for conventional and up to $20 \mathrm{~nm}$ for super resolution fluorescence microscopy under practical conditions ${ }^{1,2}$. EM provides around $1 \AA$ resolutions ${ }^{3}$, but conventional sample preparation requires dehydration, metal staining to enhance image contrast, and embedding in a mounting substance, such as resin, for transmission electron microscopy (TEM) ${ }^{4}$. To preserve biological samples in a more native-like environment, cryoEM techniques can be used ${ }^{5,6}$. The samples are rapidly 
frozen into amorphous ice, and, if needed, sectioned. Another option is freeze-fracturing $\mathrm{EM}^{7}$.

EM techniques for studying membrane proteins within intact cells in their native, liquid state have emerged in the past decade $^{8,9}, 10,11$. A spatial resolution of $2 \mathrm{~nm}$ was achieved on quantum dot (QD) labeled membrane proteins in whole cells grown on a SiN membrane and enclosed by a layer of graphene ${ }^{9}$.

Here, details of a protocol for protein labeling and graphene coating $^{9,12}$ are described. The goal of this protocol is to analyze the spatial distribution of HER2 in the membrane of whole, fixed cells, while preserving the cells in a hydrated state. Coating with graphene prevents drying of the cells in vacuum, and also reduces radiation damage ${ }^{13}$. This method provides information about labeled membrane proteins within the intact plasma membrane, but the method is not useful for studying the cellular ultrastructure as is usually done with EM.

Graphene is the thinnest nanomaterial known, and consists of a single carbon atom thick crystalline sheet arranged in a honeycomb lattice ${ }^{14}$. It has unique properties including high flexibility and mechanical strength. Recent research has shown that defect-free graphene is impermeable to gases and liquids, but defects allow hydrogen permeation ${ }^{15}$. This leakage can be reduced by using multilayer graphene as used here. Bilayer graphene has recently shown to be useful as a support for cryo-EM samples, improving the homogeneity of the thin ice layer compared to graphene oxide where only nonuniform layers can be formed ${ }^{16}$. Graphene was also shown to reduce beam damage of biological samples during liquid-phase transmission electron microscopy ${ }^{13,17}$. As an exemplary experiment, HER2 expressed in the mammalian breast cancer cell line SKBR3 was labeled with QDs ${ }^{18}$ and its spatial distribution recorded using STEM. Cells were seeded on a Si microchip with an electron transparent SiN membrane ${ }^{19}$. The microchips were chosen as a support as they are robust, compatible with LM and EM, and the entire labeling procedure can be performed directly on the microchip $^{19}$. After cell attachment, HER2 was labeled with a two-step labeling protocol ${ }^{20}$. First, a biotinylated anti-HER2 antibody mimetic compound ${ }^{21}$ was attached to HER2. The cells were then chemically fixed to prevent label-induced receptor clustering, and to increase the stability of the cellular ultrastructure. Streptavidin-coated QDs were subsequently linked to the HER2-antibody mimetic complex. The bright fluorescence signal and the electron-dense core of the QDs allowed correlative fluorescence- and electron microscopy $(\text { CLEM })^{20}$. CLEM is especially useful because cellular regions of interest for STEM analysis can be selected from the overview fluorescence microscopy images highlighting the localization of HER2 on the cells. Cells were analyzed by fluorescence microscopy to identify cellular regions with high HER2 levels. Thereafter, a 3-5 layer thick sheet of graphene was transferred onto the cells for coating ${ }^{9}, 22$. Subsequently, the sample was mounted in an EM specimen holder. STEM data was acquired using the annular dark field (ADF) detector, providing information about the spatial distribution of HER2 on the cell surface relative to the cell surface location, but giving no information about the ultrastructure of the cell. To determine the stability of the sample under electron beam irradiation, the samples were examined at increasing dose $(D)$ in an image series. The difference between graphene-coated and non-coated samples was investigated. Several kinds of radiation damage were evaluated.

The protocol described here uses the HER2 overexpressing mammalian breast cancer cell line SKBR3 as a model system for targeting HER2 ${ }^{23}$. The protocol includes the preparation of one graphene-coated sample, and one similar sample but 
without graphene coating for comparison. The experiment is prepared in duplicate since the SiN window may break once every while, and to obtain an experimental duplicate in most cases. The overall yield of the method is high meaning that microchips with graphene covered cells are usually obtained with an exceptional error even though not the entire $\mathrm{SiN}$ window may be covered with graphene in all cases. Duplicates are not described in the protocol.

The labeling protocol (steps 1-5) is comparable to the protocol of the labeling of the epidermal growth factor receptor in COS7 fibroblast cells published previously ${ }^{24}$; details in that paper are referred to regarding the handling of the microchips, and the usage of the well plates. The following protocol is optimized for labeling HER2, graphene coating ${ }^{9}$, and examining the radiation tolerance of the sample.

\section{Protocol}

\section{Cleaning of microchips and coating with poly-L- lysine (PLL) and fibronectin-like protein (FLP)}

1. Place two microchips with a SiN membrane $(2.0 \times 2.6$ $\mathrm{mm}$ ) in $50 \mathrm{~mL}$ of acetone. Handle the microchips carefully with the flat side facing up. Avoid breaking the edges by using flat-beak tweezers. Avoid touching the top surface of the microchips when handling with tweezers to prevent breakage of the SiN window.

NOTE: One may also use polytetrafluoroethylene coated or carbon tip tweezers to prevent damage of the microchips.

2. Wash the chips for 2 min by carefully shaking the beaker, watching the microchips do not flip over.

3. Transfer the microchips to $50 \mathrm{~mL}$ of ethanol and wash for 2 min by carefully shaking the beaker. Ensure that the transfer is quick so that the excess acetone does not dry in.

4. Wash the microchips with $50 \mathrm{~mL}$ of water for $10 \mathrm{~min}$.

5. Dip the microchips in a freshly prepared beaker of $20 \mathrm{~mL}$ of ethanol.

6. Place microchips onto a cleanroom tissue for drying.

7. Plasma clean the microchips with $11.5 \mathrm{sccm} \mathrm{O} 2$ and 35 sccm Ar at 70 mTorr and radio frequency (RF)-target of $50 \mathrm{~W}$ for $5 \mathrm{~min}$

8. Place the microchips in a laminar flow hood for sterile cell work.

9. Prepare a solution of $0.01 \%$ PLL in water. Prepare a solution of $15 \mu \mathrm{g} / \mathrm{mL}$ FLP in phosphate-buffered saline (PBS).

10. Prepare a 24 well plate under the laminar flow hood and fill 5 wells individually with $1 \mathrm{~mL}$ of the solutions in the following order: well 1 -PLL; well 2 - water; well 3 - water; well 4 - FLP; well 5 - PBS and well 6 - PBS.

NOTE: Conduct washing steps by dipping a microchip into the indicated 24 or 96 well for a few seconds using tweezers. Perform incubation steps by incubating the microchips in the 24 or 96 well in the indicated solution for indicated time and temperature. Transfer the microchips to another well within a few seconds.

11. Incubate the microchips in PLL solution for $5 \mathrm{~min}$. Then wash the microchips in water twice.

12. Incubate the microchips in FLP for $5 \mathrm{~min}$. Then wash the microchips in PBS twice.

13. Transfer both microchips to wells (one for each microchip) of a new 96 well plate prefilled with $50 \mu \mathrm{L}$ of serum-free medium for cell seeding. 
14. Incubate the microchips at $37{ }^{\circ} \mathrm{C}$ and $5 \% \mathrm{CO}_{2}$ until the cell suspension is prepared.

\section{Seeding cells on SiN membrane microchips}

1. Set up all supplies and equipment in a laminar flow hood to ensure sterile working.

2. Wash the breast cancer cell line, SKBR3, in a cell culture flask with growth medium once. Use Dulbecco's modified eagle medium (DMEM) containing $10 \%$ fetal calf serum (FCS), and $1 \%$ non-essential amino acids (NEAAs) as growth medium.

3. Incubate the cells with $1 \mathrm{~mL}$ of the cell detachment solution until the cells detached from the flask.

4. Add $5 \mathrm{~mL}$ of growth medium to the detached cells in the flask. Transfer this suspension to a centrifuge tube.

5. Pipette $20 \mu \mathrm{L}$ of the cell suspension into a hemocytometer to obtain the cell concentration. Use the following formula. cell concentration $=\frac{\text { total cell number of counted quadrants }}{\text { number of counted quadrants }} \times 10^{4}$.

6. Prepare a dispersed cell suspension of $2.5 \times 10^{5}$ cells/ $\mathrm{mL}$. Calculate the needed amount of the prepared cell suspension by:

amount needed $=\frac{2.5 \times 10^{5} \text { cells } / \mathrm{mL}}{\text { cell concentration }} \times$ desired volume and fill up with growth medium to the desired volume.

7. Add $100 \mu \mathrm{L}$ of the cell suspension to the two wells of a 96 well plate containing the PLL and FLP coated microchips with the SiN membrane facing up and $50 \mu \mathrm{L}$ of serum-free medium so that each well contains 25,000 cells.

8. Incubate the plate at $37^{\circ} \mathrm{C}$ and $5 \% \mathrm{CO}_{2}$ for $5 \mathrm{~min}$ to wait for the cells to attach to the microchip.

NOTE: At this point, cells can detach from the microchip because they have not adhered yet.
9. Check the density of the cells on the microchip with an inverted microscope. Make sure cells cover the window with sufficient space to flatten out and adhere (see Figure 1A).

NOTE: More cells can be added at this point if necessary.

10. Transfer the microchips into new wells containing $200 \mu \mathrm{L}$ of growth medium and incubate at $37{ }^{\circ} \mathrm{C}$ and $5 \% \mathrm{CO}_{2}$ overnight.

11. In the afternoon of the next day, transfer both microchips to serum-free medium (serum starvation medium) if cells have flattened out and adhered to a visually inspected confluency (i.e., the fraction of the window area covered with cells) of about 2/3 (see Figure 1B). Change to serumfree medium as needed to bring the cells in a defined starting condition as needed for comparison between different experiments ${ }^{25}$.

12. Incubate at $37^{\circ} \mathrm{C}$ and $5 \% \mathrm{CO}_{2}$ overnight.

NOTE: Be aware that cell amounts may differ according to growth rates and cell morphology for other cell lines.

\section{HER2 labeling and fixation}

1. Prepare the solutions as described in Supplementary Table 1.

1. Work under the laminar flow hood to ensure sterile working. Use a 96 well plate referred to as labeling plate I, and fill 6 wells per microchip with $200 \mu \mathrm{L}$ of the labeling plate I solutions: PBS/BSA, PBS/BSA/ GS, antibody mimetic, PBS/BSA, PBS/BSA, PBS/ BSA. Use one row (one letter per row, e.g., A1 to A6) of the well plate per microchip. Warm the labeling plate to $37^{\circ} \mathrm{C}$. 
2. Under a fume hood, prepare a 24 well plate referred to as fixation plate, and fill 8 wells per microchip with 500 $\mu \mathrm{L}$ of the fixation plate solutions: $\mathrm{CB}, \mathrm{FA}, \mathrm{CB}, \mathrm{PBS}$, PBS, PBS, PBS/glycine, PBS/BSA.

CAUTION: CB is acutely toxic by inhalation or oral ingestion and is hazardous to waters. Work under the fume hood with appropriate protection and dispose $\mathrm{CB}$ according to the safety data sheet (SDS). FA is corrosive and harmful for skin and health. Work under the fume hood and refer to the SDS for information about handling and disposal.

3. Prepare a 96 well plate referred to as labeling plate II and fill 4 wells per microchip with $200 \mu \mathrm{L}$ of the labeling plate II solutions: QDs, PBS/BSA, PBS/BSA, PBS/BSA.

NOTE: Here, a 24 well plate is used to better see the microchips in the wells. To use less antibody mimetic (step 3.2) and QDs (step 3.4), use 96 well plates for these steps.

2. Label HER2 in the cells.

1. Once these plates are ready, start labeling by placing the microchips into the wells of the first row of labeling plate 1.

2. Wash the microchips with PBS/BSA in the 96 well plate marked as labeling plate I.

3. Block unspecific sites to prevent unspecific binding of the antibody mimetic by incubating with PBS/BSA/GS for $5 \mathrm{~min}$ at $37{ }^{\circ} \mathrm{C}$ and $5 \% \mathrm{CO}_{2}$.

4. Incubate with $200 \mathrm{nM}$ antibody mimetic for $10 \mathrm{~min}$ at $37^{\circ} \mathrm{C}$ and $5 \% \mathrm{CO}_{2}$.

5. Wash microchip three times in PBS/BSA.
1. Transfer the microchips to the 24 well fixation plate in the fume hood.

NOTE: No sterile work is needed from here.

2. Wash once with $\mathrm{CB}$ for a few seconds.

3. Fix cells with $3 \% \mathrm{FA}$ for $10 \mathrm{~min}$.

4. Wash once with $\mathrm{CB}$ and three times with PBS.

5. Block free aldehyde groups of FA by incubating with PBS-glycine for $2 \mathrm{~min}$.

6. Wash microchips with PBS-BSA once.

4. Attach the QDs.

1. Move the microchips to the 96 well labeling plate II.

2. Incubate with $20 \mathrm{nM}$ QDs for $12 \mathrm{~min}$.

3. Wash the microchips with PBS/BSA twice.

4. Store the microchips in a well containing PBS/BSA.

\section{Light microscopy of the fixed cells}

1. Prepare a $3.5 \mathrm{~cm}$ diameter glass bottom dish with $2 \mathrm{~mL}$ of PBS/BSA solution.

2. Take the first microchips, place it upside down (cells facing down) into the glass bottom dish and place the dish in the fluorescence microscope. Lower the microchip slowly into the liquid to prevent damage on the cells.

3. Acquire differential interference contrast (DIC) and fluorescence images of every microchip with a $40 x$ objective and the appropriate fluorescence channel.

NOTE: Here, an excitation wavelength of $540-580 \mathrm{~nm}$ and an emitting wavelength of $607-683 \mathrm{~nm}$ is used to detect QD655.

4. Repeat the procedure for the second microchip.

3. Fix the cells. 


\section{Post-fixation}

1. Perform all steps under the fume hood.

2. Fill 6 wells per microchip of a 96 well plate with $200 \mu \mathrm{L}$ of the post-fixation solutions:

CB, GA, CB, PBS, PBS, PBS.

CAUTION: GA is hazardous to waters, harmful for skin, respiratory system, and eyes. Work under the fume hood and refer to the SDS for information about handling and disposal.

3. Place both microchips in their respective wells with the cells facing up.

4. Wash once with $\mathrm{CB}$.

5. Fix cells with $2 \%$ GA for 10 min.

6. Wash once with $\mathrm{CB}$.

7. Wash three times with PBS/BSA.

NOTE: The first fixation step with FA already fixes the biological structure but a reduced level of membrane protein diffusion may still occur, possibly leading to label induced clustering due to the presence of multiple streptavidins per QD. Keep the time of the experimental steps from FA fixation to GA, therefore, as short as possible to minimize the diffusion of the proteins in the membrane.

8. Store in PBS/BSA to prevent osmotic shocks and $0.02 \%$ sodium azide $\left(\mathrm{NaN}_{3}\right)$ to prevent bacterial growth at $4{ }^{\circ} \mathrm{C}$ until graphene coating in a new well plate. Seal well plate with paraffin film to prevent drying. The microchips and cells are stable up to 2 weeks when stored at $4{ }^{\circ} \mathrm{C}$.

CAUTION: $\mathrm{NaN}_{3}$ is hazardous to waters and acutely toxic by oral ingestion, for skin and the respiratory system.
Work under the fume hood and refer to the SDS for information about handling and disposal.

\section{Cleaning and transferring graphene onto salt crystals}

1. Remove poly(methyl methacrylate) (PMMA)-graphene from PMMA-graphene-on-polymer (Figure 2A).

1. Pipette a few droplets of water on the polymer around the PMMA-graphene.

NOTE: Ensure that the PMMA-graphene easily comes off the supporting polymer as it floats on the water surface.

2. Immerse the PMMA-graphene-on-polymer into water with an angle of $30-45^{\circ}$ to release the PMMAgraphene.

NOTE: The polymer should only be slightly wetted. Pipetting too much water on the polymer will lift up and possibly fold the PMMA-graphene.

2. Etch copper-based contaminants using a sodium persulfate solution (Figure 2B).

NOTE: Commercial graphene grown on copper foil often contains sub-micrometer copper residues, which can be removed with a copper etchant solution ${ }^{22}$

1. Prepare a $50 \mathrm{~mL}$ solution of $0.42 \mathrm{M}$ sodium persulfate in water

2. Transfer PMMA-graphene into the sodium persulfate solution with the graphene side down using a standard glass slide. PMMA-graphene will float on top of the solution.

3. Leave the PMMA-graphene in the sodium persulfate solution overnight.

4. Remove the PMMA-graphene from the sodium persulfate solution and place it on top of clean water 
using a glass slide. Let it float on the water for half an hour.

5. Repeat the previous step a total of three times to remove all sodium persulfate residues from the PMMA-graphene.

3. Transfer the PMMA-graphene onto a sodium chloride $(\mathrm{NaCl})$ crystal.

1. Prepare a saturated solution of $\mathrm{NaCl}$ in water in a Petri dish.

2. Transfer the PMMA-graphene on top of the $\mathrm{NaCl}$ solution with the graphene side down using a glass slide.

3. Hold a $\mathrm{NaCl}$ crystal with tweezers and pick up the floating PMMA-graphene.

NOTE: The size of the $\mathrm{NaCl}$ crystal should be slightly larger than the size of the PMMA-graphene to avoid folding the protruding graphene at the edge of the salt or contacting the graphene with tweezers. In these experiments $\mathrm{NaCl}$ crystal of $12 \mathrm{~mm} \times 12 \mathrm{~mm} \times 0.5 \mathrm{~mm}$ is used for picking up a $10 \mathrm{~mm} \times 10 \mathrm{~mm}$ graphene sheet and supporting it afterwards.

4. Hold the PMMA-graphene-on-salt vertically for 2 min to let excess water flow out.

5. Let the PMMA-graphene-on-salt dry at room temperature for 30 min and bake it in an oven at 100 ${ }^{\circ} \mathrm{C}$ for 20 min to completely remove water.

4. Remove PMMA using an acetone wash (Figure 2C).

1. Preheat acetone in a glass Petri dish to $\sim 50{ }^{\circ} \mathrm{C}$ on a hotplate in the fume hood. Watch the temperature carefully to avoid fire.
2. Immerse the PMMA-graphene-on-salt into the Petri dish filled with acetone and leave it to dissolve the PMMA for $30 \mathrm{~min}$.

3. Repeat the previous step a total of three times with new, clean acetone.

4. Let the graphene-on-salt air-dry thoroughly before using it for sample preparation.

\section{Graphene Coating}

NOTE: The graphene coating procedure is schematically shown in Figure 3A.

1. Wash one microchip prepared with fixed cells and labeled HER2 in pure water to remove any residues of salt from the buffer. Place the microchip on a filter paper. The cells are visible as dark spots (Figure 3B).

2. Cut the multilayer graphene on $\mathrm{NaCl}$ crystal into a piece that fits the SiN window of a microchip using a razor blade.

3. Prepare a beaker of pure water and remove the graphene from the $\mathrm{NaCl}$ crystal by tilting the crystal about $45^{\circ}$ angle with respect to the water surface and touching the water. The graphene will float onto the water surface (Figure $3 \mathrm{C})$.

4. Catch the graphene with a metal loop from the surface of the water. The graphene will float within the droplet below the loop (Figure 3D).

5. Touch the upper surface of the microchip with the lower loop surface. The microchip will stick to the metal loop. The graphene can be seen on top of the microchip (Figure 3E).

6. Under a stereomicroscope, use filter paper to remove the remaining water from the microchip, so that the graphene covers all cells on the SiN window. 
NOTE: The graphene will move when blotted. Make sure the graphene stays on the top of the window by touching the microchip just with the edge of the filter paper.

7. Use tweezers to remove the microchip from the metal loop and place it onto a filter paper. The graphene is visible as a purple shimmer on the microchip (Figure 3F).

8. Transfer the microchip from the paper to a compartment Petri dish.

9. Pipette a droplet of water into one of the free compartments and close the lid to provide a watersaturated atmosphere.

10. Seal the compartment dish with paraffin film and store in the fridge at $4{ }^{\circ} \mathrm{C}$ if required for further measurements.

\section{STEM}

1. Adjust the STEM at $200 \mathrm{kV}$ beam energy using an alignment/test sample for a probe size of at least $0.2 \mathrm{~nm}$ by adjusting the condenser lenses, a probe current $I=$ $180 \mathrm{pA}$ (information about the probe current for different microscope settings is provided by the manufacturer within $5 \%$ accuracy) and a beam convergence semi-angle of $13.2 \mathrm{mrad}$ by inserting an aperture. Set the ADF STEM detector opening semi-angle range (inner and outer) to 68-280 mrad by adjusting the projector lens settings. Set the STEM image size to $2048 \times 2048$ pixels, and the pixel dwell time $t=6 \mu \mathrm{s}$.

2. Load the microchip with graphene-coated cells in a standard specimen holder for TEM in such a way that the cells are facing up.

3. Load the holder into the electron microscope.

4. Acquire an overview picture at magnification $(M)=800 \mathrm{x}$

(Figure 4) using the settings in step 8.1.
5. Identify a region of interest on a cell.

6. Image the QDs with an ADF detector at $M=80,000 x$ at a pixel size $d=1.3 \mathrm{~nm}$ (Figure 4 ) using the settings in step 8.1.

7. Acquire an image of the area after exposure with a lower magnification (here, $M=50,000 x$ ) to show the exposed area (Figure 4).

8. Calculate the electron dose

$D=\frac{l \times t}{e \times d^{2}}$

where $e$ is the elementary charge. With the settings given in the above,

$D=\frac{1.75 \times 10^{-10} \times 6 \times 10^{-6}}{1.6 \times 10^{-19} \times\left(1.3 \times 10^{-9}\right)^{2}}=39 \mathrm{e} / \AA^{2}$

and an error of $5 \%$.

9. Acquire 20 images for a dose series with the same settings, but with $t=60 \mu$ s resulting in an accumulated dose of

$D=20 \times(39 \pm 2)=(7.8 \pm 0.4) \times 10^{3} e^{-} / \AA^{2}$.

10. To confirm the presence of graphene, switch the microscope to TEM at $M=1,200 \mathrm{x}$, select a region near a cell, and switch to diffraction mode. Record a diffraction pattern at an exposure time of $0.5 \mathrm{~s}, 2048 \times 2048 \times 3$ pixels, and a selected area aperture of $50 \mu \mathrm{m}$ (Figure 4).

11. At the end of the session, remove the sample from the microscope, place the microchip back into the compartment dish, seal the dish with paraffin film, and store it in the fridge at $4{ }^{\circ} \mathrm{C}$ if required for further measurements.

12. Select the second microchip prepared in the same way as the first microchip but without graphene coating. 
13. Repeat steps 8.2-8.11 but now for this sample. Record a diffraction pattern with the same settings as in the above, as comparison (Figure 4).

\section{Analysis}

NOTE: For automated detection of QD positions in a STEM image, the analysis uses a plugin of local design for Image $(\mathrm{NIH})$, as described elsewhere ${ }^{20}$. The plugin is available upon request.

1. The software automatically applies the following steps to detect particles in a STEM image:

1. Apply a Gaussian filter to reduce pixel noise.

2. Apply a Fast Fourier Transform (FFT) bandpass filter to obtain nanoparticles only.

3. Set a threshold to binarize the image.

4. Use a particle diameter of $10 \mathrm{~nm}$ with a tolerance factor of 2 for particle detection.

5. Detect particle positions.

2. Measure the center-to-center distance between ten pairs of QDs per series. Here, 10 distances with varying size were measured per series.

3. Calculate the relative change in particle distance by comparing each particle distance to the distance in the first image using:

Relative change $=\left(\frac{\text { Distance in image }}{\text { Distance in first image }} \times 100\right)-100$.

\section{Representative Results}

Figure 1A shows cells seeded in such a way that the window is covered but with sufficient space to allow them to flatten out and adhere, leading to confluency of about $2 / 3^{\text {rd }}$ (Figure 1B). In case too many cells are seeded on a microchip (Figure
1C), there is insufficient space for all cells to adhere to the microchip. Figure 1D shows the same microchip after $24 \mathrm{~h}$. More than half of the cells didn't flatten. On the other hand, if too few cells are seeded (Figure 1E), the SiN window will end up with a large empty space after $24 \mathrm{~h}$ as seen in Figure 1F. Figure 1G shows the DIC image of the cells in Figure 1A and Figure 1B. Figure $1 \mathrm{H}$ shows the corresponding fluorescence image false colored in yellow indicating successful labeling of HER2.

Representative STEM data is shown in Figure 4. Graphenecoated (left column) and non-coated (right column) SKBR3 cells were investigated. Figure 4A,B show $M=800$ x overview images of the cells on the window. The areas shown as insets were imaged at $M=80,000 x$ during the dose series, see Figure 4C,D. The QDs are visible as bright spots here. Figure 4E and Figure 4F show $M=50,000 x$ magnified images acquired at the locations of the rectangles in Figure 4C,D. Both images were recorded after the acquisition of a dose series with $D=(7.8 \pm 0.4) \times 10^{3} \mathrm{e}^{-} / \AA^{2}$. The dose series was recorded at $M=80,000 x$. The exposed areas can be recognized as rectangles, whereby the rectangle was clearly visible for the non-coated sample (Figure 4F).

To verify the presence of graphene, diffraction patterns of areas without cells, but with or without graphene on the $\mathrm{SiN}$ window were acquired. The hexagonal structure of the graphene was observed in the diffraction pattern of the graphene-coated sample (Figure 4G), while it was absent for the non-coated sample (Figure $\mathbf{4 H}$ ). The diffraction pattern of single crystal graphene will have six-fold symmetry due to highly ordered hexagonal structure of graphene. So, the hexagonal structure indicates the presence of graphene on the samples. 
To investigate the effect of electron beam illumination on the sample, STEM images were acquired in an image series with an accumulating electron dose. Representative results for non-coated and graphene-coated samples are shown in Figure 5A-D and Figure 5E-G, respectively. All data were acquired at the edge of the cell, where the cell is the flattest, and the observed structure is thus the closest to the $\mathrm{SiN}$ membrane. The exposure of a non-coated samples led to bright structures appearing on the cell surfaces at $D=(1.9$ $\pm 0.1) \times 10^{3} \mathrm{e}^{-} / \AA^{2}$ (Figure 5B). These structures became larger with higher doses, so they were clearly visible in the last image of the series at $D=(7.8 \pm 0.4) \times 10^{3} \mathrm{e}^{-} / \AA^{2}$ (Figure 5C,D). These spots did not appear on any of the graphenecoated samples (Figure 5E-G).

Additional microchips were prepared and examined using the protocol described in the above. Six coated and seven non-coated samples were investigated in total. Two out of seven non-coated samples showed these artifacts. None of the coated samples showed any additional bright spots.

As another measure of radiation damage, the distance between QDs was examined. If structural damage were to occur, one would expect distances between QDs to change. Changes in distances were measured for different pairs of QDs with accumulating $D$ for a range of pair distances. Figure $5 \mathrm{H}$ shows that the relative change of the particles for noncoated samples stayed below $1.3 \%$ on average, while the average relative distance remained below $0.8 \%$ for the coated samples. One can, therefore, conclude that the graphene coating stabilized the sample but the dried-in samples without graphene coating was also remarkably stable. 

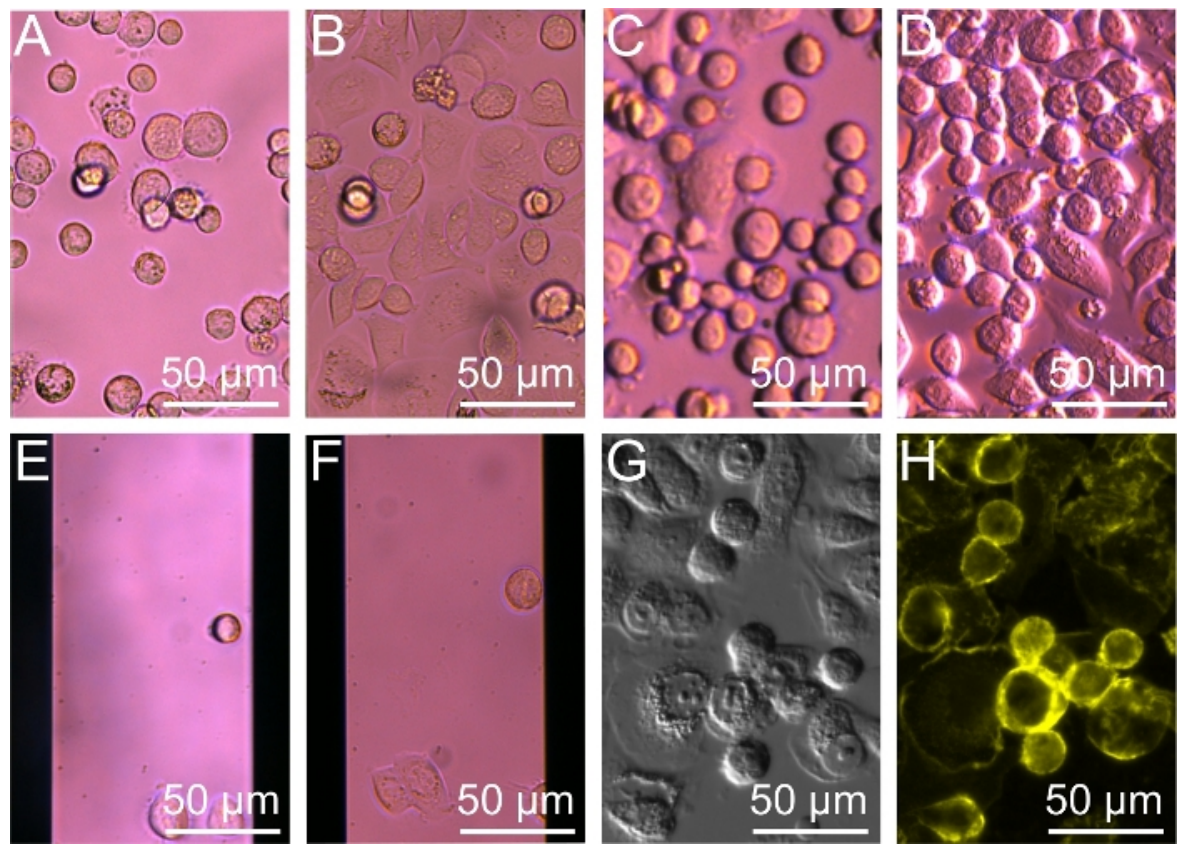

Figure 1: Cell seeding on a SiN window of a silicon microchip and HER2 labeled. (A) Exemplary image of a SiN window region with SKBR3 cells 5 min after seeding on the microchip. (B) The same cells spread on the same SiN window after 24 h. (C) SKBR3 cells on a Si microchip 5 min after seeding. (D) Same cells as in (C) after $24 \mathrm{~h}$. Cells didn't flatten properly as there were too many cells on the chips upon seeding. (E) Cells on a microchip 5 min after seeding. (F) Only few cells were visible on the window because too few cells were seeded on the microchip. (G) DIC image of the same SKBR3 cells after QD labeling of HER2. (H) Overlay image of DIC and corresponding fluorescence image of labeled SKBR3 cells with HER2QD655 (false colored in yellow). Please click here to view a larger version of this figure. 
A

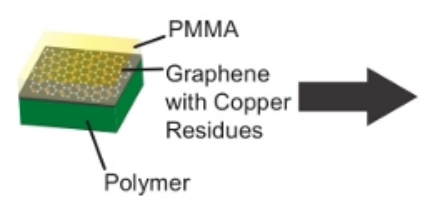

B
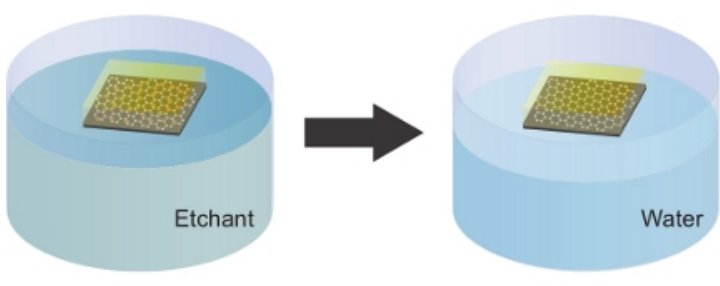

C

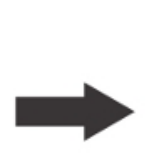

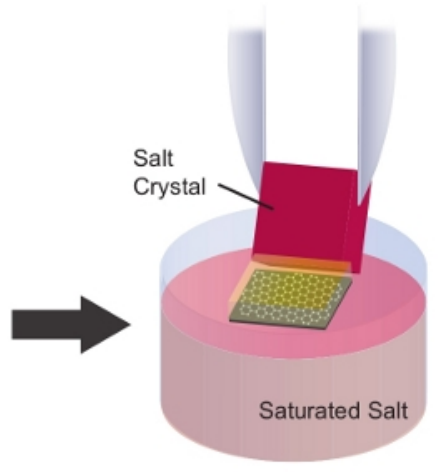

Salt Crystal
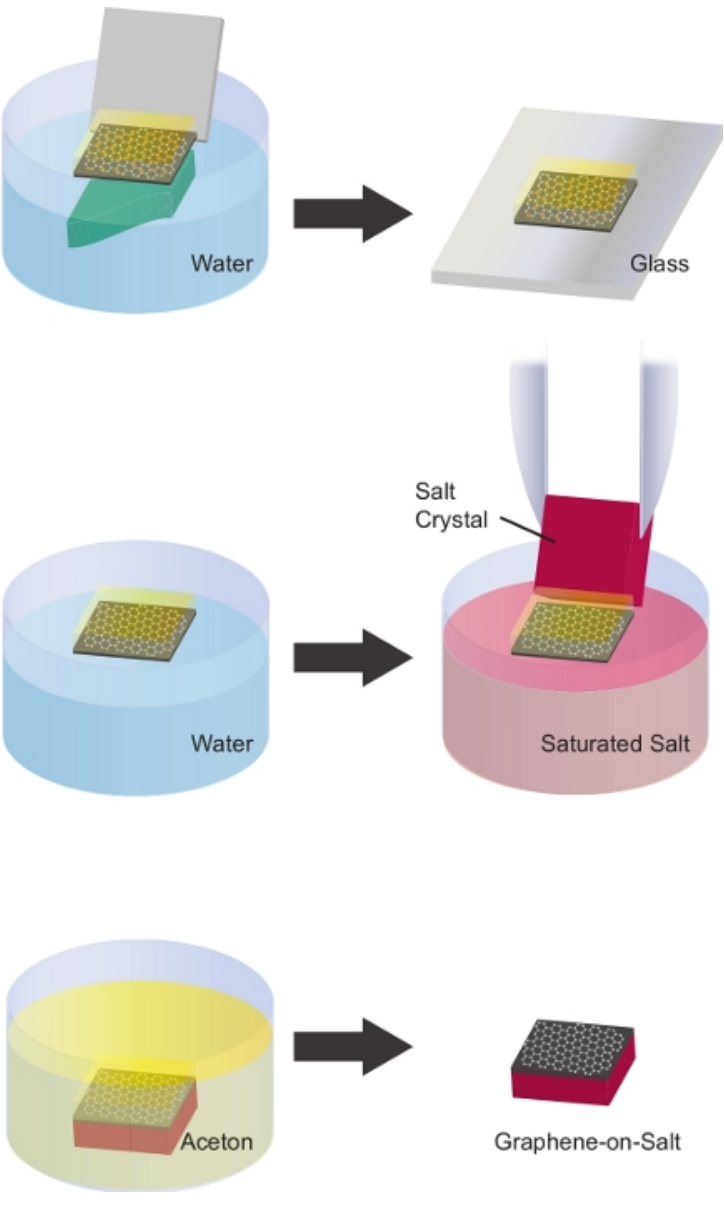

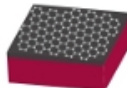

Graphene-on-Salt

Figure 2: Cleaning and transferring of graphene onto $\mathrm{NaCl}$ crystals. (A) PMMA-graphene-on-polymer is immersed in pure water to release PMMA graphene. PMMA-graphene can be caught with a glass slide. (B) Copper-based contaminations were etched using sodium persulfate solution. To clean the graphene, it was transferred to a beaker containing deionized water. These steps were repeated 3 times. The PMMA-graphene was then transferred to saturated solution of $\mathrm{NaCl}$ in pure water. A NaCl crystal was used to pick up the graphene from the salt solution. (C) The PMMA-graphene on $\mathrm{NaCl}$ crystal was dried out for $30 \mathrm{~min}$ at room temperature. PMMA was removed by incubating the block in acetone for 30 min. Please click here to view a larger version of this figure. 

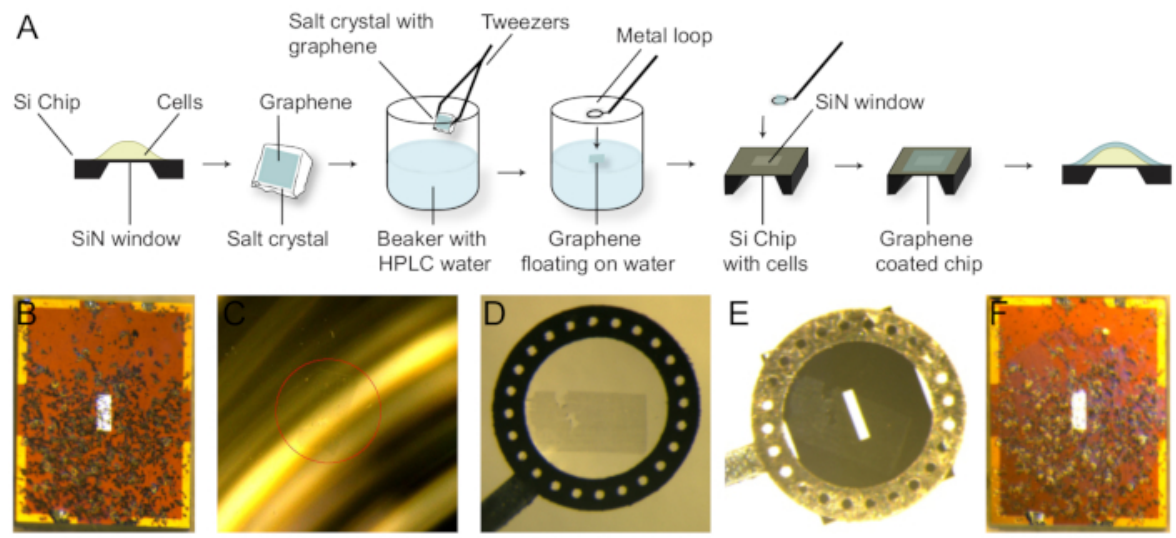

$\mathrm{E}$

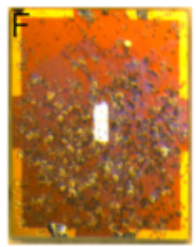

Figure 3: Graphene coating of cells seeded on a Si microchip. (A) Procedure of graphene coating. Graphene on NaCl crystal is released onto the water surface. The graphene piece is then caught with a metal loop and transferred onto the Si microchip. (B) Microchip $(2.0 \times 2.6 \mathrm{~mm})$ with a SiN window of dimensions $400 \times 160 \mu \mathrm{m}$ without graphene. SKBR3 cells were visible as dark spots. (C) Graphene (red circle) floating on the surface of a beaker filled with water. (D) Graphene caught with a metal loop. (E) The microchip attached to the water droplet so that the graphene was on top of the SiN window. (F) Microchip after graphene coating. The graphene was visible as a purple shimmer. Please click here to view a larger version of this figure. 

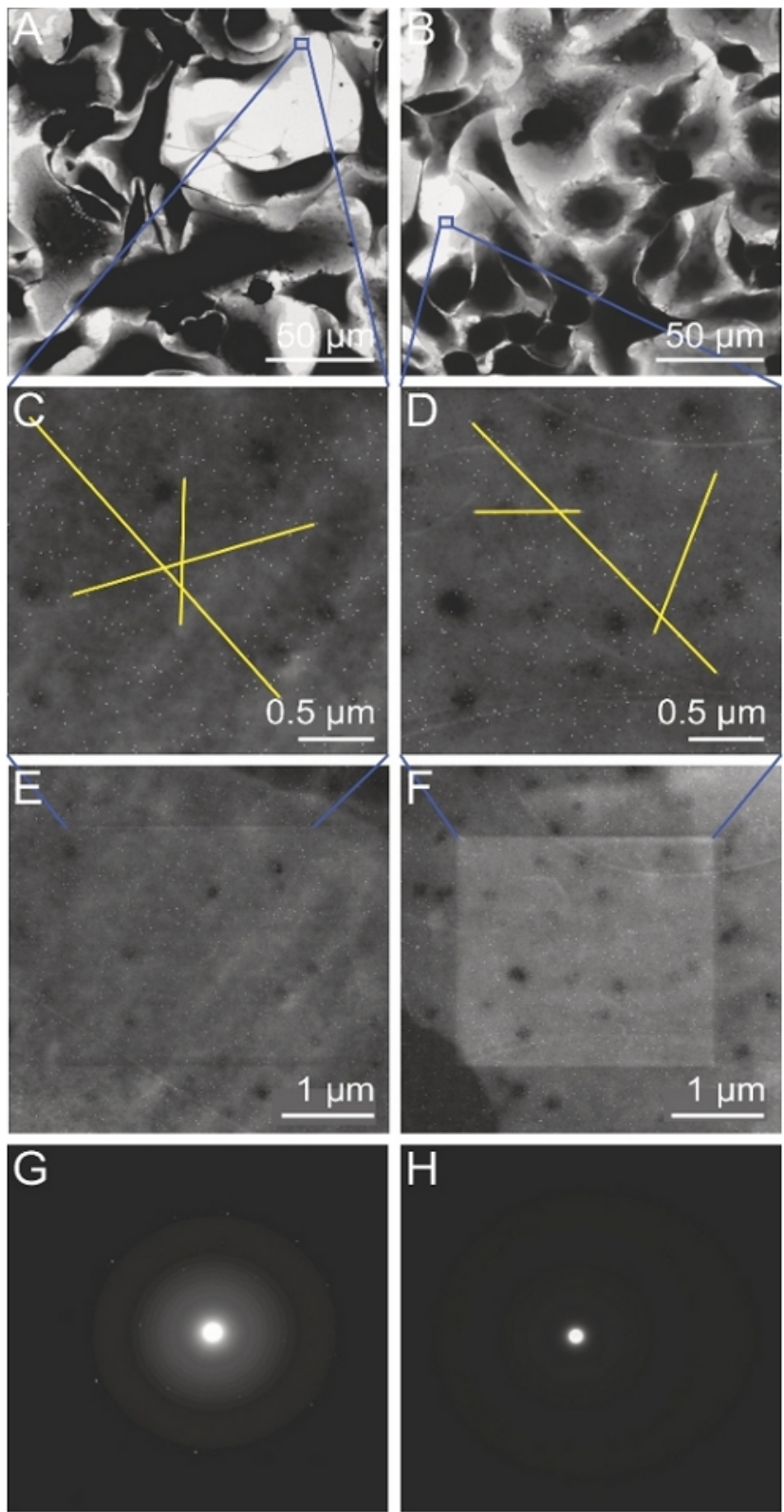

Figure 4: STEM of graphene coated and non-coated SKBR3 cells on SiN window. (A) STEM image of a graphene coated sample acquired at a $M=800 x$. (B) STEM image of a non-coated sample acquired at $M=800 x$. (C) $M=80,000 x$ image recorded at the position of the blue rectangle in A. Yellow lines represents examples distances measured within particle. (D) $M=80,000 x$ image recorded at the position of the blue rectangle in B. Yellow line represents examples distances measured within particles. (E) $M=50,000 x$ image of the same region as $C$ exposed to $D=(7.8 \pm 0.4) \times 10^{3} \mathrm{e}^{-} / \AA^{2}$. (F) $M=50,000 x$ image of the same region as $\mathbf{D}$ and exposed to $D=(7.8 \pm 0.4) \times 10^{3} \mathrm{e}^{-} / \AA^{2}$. The exposed area were clearly seen. (G) Diffraction pattern of a graphene-coated sample from an area without cells. The six-fold symmetry of the graphene 
is visible as bright spots. (H) Diffraction pattern of a sample without graphene showing no 6-fold bright spots. Please click here to view a larger version of this figure.
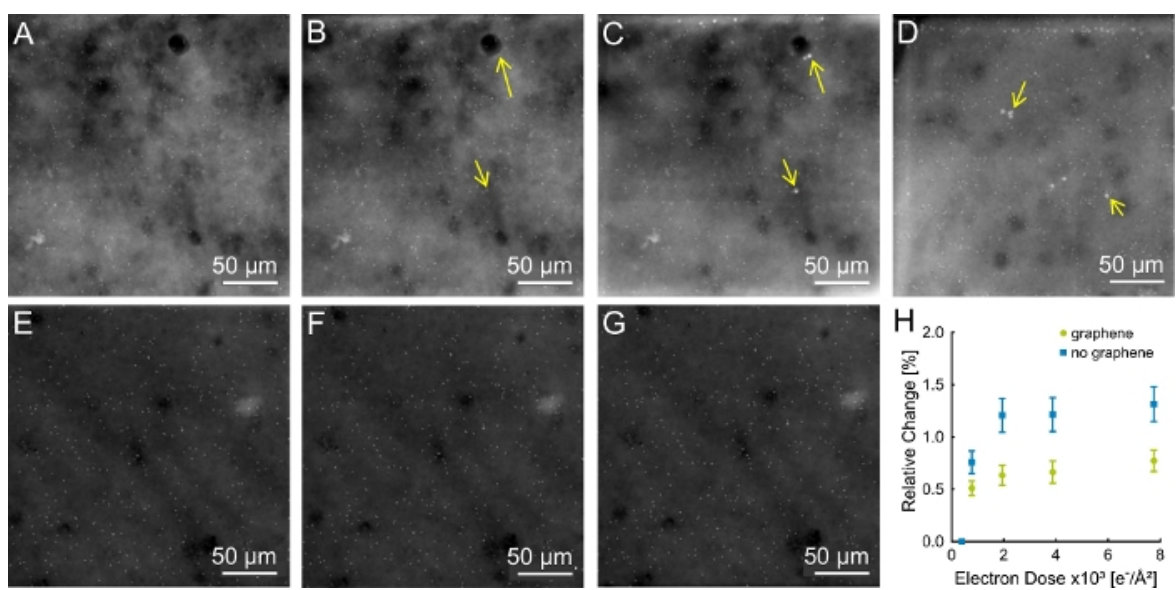

Figure 5: Artifacts arising on samples without graphene coating. (A) First images of regions on samples without graphene coating acquired at $M=80,000 \mathrm{x}$, and exposed to $D=(0.39 \pm 0.02) \times 10^{2} \mathrm{e}^{-} / \AA^{2}$. (B) Images with the first artifacts arising at $D=(1.94 \pm 0.1) \times 10^{3} \mathrm{e}^{-} / \AA^{2}$ (yellow arrows). (C, D) Last image of the series acquired with $D=(7.8 \pm 0.4) \times 10^{3}$ $\mathrm{e}^{-} / \AA^{2}$. Artifacts were visible as bright spots. (E-G) Graphene coated samples without arising artifacts. (E) $D=(0.39 \pm 0.02)$ $\times 10^{2} \mathrm{e}^{-} / \AA^{2}(\mathbf{F}) D=(1.94 \pm 0.1) \times 10^{3} \mathrm{e}^{-} / \AA^{2}(\mathbf{G}) D=(7.8 \pm 0.4) \times 10^{3} \mathrm{e}^{-} / \AA^{2}$. (H) Relative change in particle distances for graphene coated and non-coated samples. Two of the non-coated samples showing artifacts, one of which shown in (A-C), and the last image of the second sample in $D$, as well as three coated samples were analyzed (one is shown in E-G). In total, ten QD pairs were examined per sample with distances ranging between $250 \mathrm{~nm}$ and $3 \mu \mathrm{m}$. The relative change reflects the average of all measurements in one group. Please click here to view a larger version of this figure.

Supplementary Table 1: Recipes for solutions and buffers. Please click here to download this table.

\section{Discussion}

To better understand protein function, it is important to obtain information about protein locations in the plasma membrane of intact cells. Methods for obtaining this information include super-resolution fluorescence microscopy ${ }^{1,2}$. Although super-resolution microscopy has further developed over the past years, its resolution is still limited to about $20 \mathrm{~nm}$ for practical conditions of cell experiments, while typical receptor proteins have sizes in the range of 1-10 $\mathrm{nm}$. The imaging of proteins on single cell and single molecule level with sufficient resolution to visualize proteins is possible with EM. But due to sectioning, conventional EM methods typically do not leave the cell intact ${ }^{26}$, which leads to the loss of important information about the context and the spatial distribution of proteins in the plasma membrane. Methods for whole cells with cryo-TEM have been developed ${ }^{6}$, it is feasible to combine protein labeling with cryo-EM ${ }^{27}$, also cryo-STEM has been demonstrated ${ }^{28}$. However, cryo-EM workflows are optimized for studying the cellular ultrastructure and protein structure, and not so much for analyzing membrane 
protein spatial distributions. Critical point drying is another whole cell preparation method but the samples are subjected to several drying steps, and the technique is highly timeconsuming $^{29}$. Membrane proteins have also been examined via freeze fracture ${ }^{7}$. In this method, cells are fixed, frozen, and fractured. The fractured parts are replicated by carbon and platinum layers, and the biological sample is removed. The replicas can then be analyzed with $\mathrm{EM}^{30}$. Whole cell analysis is impossible with freeze fraction because information about the distribution of proteins in the membrane within context of the whole cell is lost.

The method presented here allows the cell membrane to be studied without needing to thin-slice the specimen ${ }^{9}, 31$. The cells are kept intact so that the localization of the membrane proteins is visible from the fluorescence images, which are correlated with the EM images. Studying proteins at the single cell and single molecule level within intact cells in hydrated state has shown to be possible with a resolution of $2 \mathrm{~nm}$ using STEM of QD labeled proteins using this graphene enclosure method $^{9}$. Keeping cells in their native state is crucial, as it preserves the spatial distribution of membrane proteins such that analyses are possible at the single cell and single molecule level, which is important for understanding protein functions, and developing new drugs for therapy approaches.

Another critical aspect of imaging biological samples with EM is the radiation damage of the samples caused by the electron beam. Solutions often include the reduction of the electron dose as much as possible or various coating methods, such as encapsulating the specimen between thin layers of carbon $^{32}$. Our method shows that graphene coating reduces beam-induced artifacts that emerge on the cell surface for non-coated samples. Examination of the chemically fixed, and graphene coated biological samples is possible under electron beam irradiation at $200 \mathrm{keV}$ beam energy up to $D$ $=(7.8 \pm 0.4) \times 10^{3} \mathrm{e}^{-} / \AA^{2}$ without radiation damage, such as bright spots, appearing on the sample. Compared to other EM methods that involve elaborate sample preparation, for example, staining, embedding, (cryo-) sectioning, fracturing, etc., the method described here is less time consuming. Labeling of the proteins is performed within a few hours, and graphene coating only requires about $15 \mathrm{~min}$ for trained researchers. The sample preparation is comparable with the procedures needed for fluorescence microscopy.

The protocol can be modified in some steps. The graphene on the microchip can also be air-dried to make sure that the graphene does not move when blotted with a filter paper. If the graphene is contaminated with salt, it is possible to let it float on the surface of water for about one hour to dissolve the salt and thus minimize contamination. Occurring copper or PMMA contamination on the graphene can be reduced by extending the corresponding etching steps in the protocol. Other graphene coating methods have been described where, for example, graphene-PMMA was directly deposited on cells, and the PMMA was removed by washing in acetone afterwards ${ }^{33}$. In our method, PMMA was removed before coating to avoid any possible damage to the cells caused by additional acetone washing steps. $\mathrm{NaCl}$ was chosen as a substrate here because it is flat, so it does not wrinkle the graphene, and it dissolves in water to release the graphene ${ }^{9}$. Besides, it can be cut into the desired size and no substrate residues are left on the graphene. But taking those criteria in consideration, other substrates like potassium chloride can possibly be used as well.

To reduce the chance of potential label-induced clustering, the GA fixation step can be implemented directly after FA fixation, after which all membrane proteins are immobilized. 
The first fixation step with FA already fixes the biological structure but a reduced level of membrane protein diffusion may still occur ${ }^{34}$, possibly leading to label induced clustering due to the presence of multiple Streptavidin per QD. Fixation with GA may lead to an autofluorescence signal during LM, and is, therefore, done after LM in the described protocol but can be reduced as described elsewhere ${ }^{34}$. Cacodylate buffer is quite toxic, and other fixatives can be used as well, but cacodylate is used here as it is a commonly used buffer for EM protocols, avoids precipitates, prevents the growth of bacterial and fungi, and is compatible with calcium ions that are needed to preserve the ultrastructural integrity of lipid membranes $^{35}$. If needed, osmium tetroxide can be used as additional fixation for stabilizing the lipids. This would help to enhance contrast of the cell structure, but also add another metal to the system and reduce the contrast obtained on the QDs.

The protocol described here contains many steps that require good instruction. Some training is required before handling microchips to avoid scratching the SiN surface of the microchips and to prevent breakage. As mentioned before, it is recommended to prepare microchips in duplicates as the SiN window can break from time to time. Obtaining the required number of cells on a microchip also requires some experience. Coating the cells with graphene needs some training as it can be difficult to find the right tilting angle to float graphene on water. When catching the graphene from water, it may also be difficult to see the thin graphene. As soon as the graphene is on the microchip, excess water needs to be blotted off with a filter paper. This should only be done with the tip of a filter paper such to avoid removing the graphene from the microchip.
Graphene coating prevented artifacts from appearing on the sample. But for $D<4 \times 10^{2} \mathrm{e}^{-} / \AA^{2}$ also no artifacts emerged for the non-coated sample, and artifacts appeared for 2 non-coated samples only. Thus, examinations of noncoated cells also seem possible, although it would be better to use graphene and avoid the risk of artifact formation. The composition of those artifacts can be analyzed in the future to give hints about how to prevent their formation. Regarding the structural stability of the cells only a minor improvement of the graphene coating was observed. The fixed cells were apparently stabilized in the examined thin areas, where their structure was in close proximity of the SiN membrane. What we did not examine here, however, were drying artifacts that are known to occur for cellular samples when exposed to vacuum ${ }^{4}$. Drying of the cells would lead to shrinkage of the cells so that also the QD distances would change as a consequence. For the electron dose used here, the distance of QDs of graphene-coated and non-coated samples remained stable. Further studies are needed to examine the effect of graphene coating on the cells for EM.

One limitation of this method is that the chemical fixation of the cells is necessary; therefore, no live cell experiments can be performed. But in case the labeling is not needed and cells with a higher structural stability are used, for example bacteria, then unfixed cells can be enclosed in graphene for $\mathrm{EM}^{36}$ albeit with a different electron dose tolerance. Also, the proteins are not directly detectable, so QDs are needed to visualize the proteins. The method would benefit from smaller labels. A point of discussion is whether it is good or bad that the ultrastructure is not clearly visible. Our method is similar to that of fluorescence microscopy where only selected proteins are visible ${ }^{37}$. Increasing the visibility of the ultrastructure would also add much more information to the image, and then at some point prevent detection 
of the individual label positions. Furthermore, the method described here is for one protein species, and additions of the protocol are needed to be able to label multiple proteins. Last, the method works when a small high affinity specifically binding molecule such as antibody mimetic ${ }^{21}$ or nanobody ${ }^{38}$ is available. Commonly used antibodies are much larger and would prevent the detection of the functional state of the protein subunits into oligomers.

Our method is useful for studying protein function on whole cells using EM while keeping the cells in hydrated state. It is readily possible to examine series of cells. Other type of cells and proteins can be studied as well. If protein labeling is not needed, a subset of the protocol can be used for graphene coating of wide variety of biological specimens. The ability to study whole cells is relevant in cellular research for understanding correlations of membrane protein function at the molecular level.

\section{Disclosures}

The authors have nothing to disclose.

\section{Acknowledgments}

We thank D. B. Peckys for help with the cell culture protocol, F. Weinberg for reviewing the manuscript, T. Trampert for help with the experiments and the figures, S. Smolka for help with the figures, and E. Arzt for his support through INM. This research is funded by Else Kröner-Fresenius-Stiftung.

\section{References}

1. Hell, S. W. Far-field optical nanoscopy. Science. 316 (5828), 1153-1158 (2007).
2. Sigal, Y. M., Zhou, R., Zhuang, X. Visualizing and discovering cellular structures with super-resolution microscopy. Science. 361 (6405), 880-887 (2018).

3. Williams, D. B., Carter, C. B. The Transmission Electron Microscope. Springer (2009).

4. Bozzola, J. J., Russell, L. D. Electron Microscopy Principles and Techniques for Biologists. Jones and Barlett Publishers (1999).

5. Thompson, R. F., Walker, M., Siebert, C. A., Muench, S. P., Ranson, N. A. An introduction to sample preparation and imaging by cryo-electron microscopy for structural biology. Methods. 100, 3-15 (2016).

6. Lucic, V., Leis, A., Baumeister, W. Cryo-electron tomography of cells: connecting structure and function. Histochemistry and Cell Biology. 130 (2), 185-196 (2008).

7. Carson, J. L. Fundamental technical elements of freezefracture/freeze-etch in biological electron microscopy. Journal of Visualized Experiments. (91), 51694 (2014).

8. Nishiyama, H. et al. Atmospheric scanning electron microscope observes cells and tissues in open medium through silicon nitride film. Journal of Structural Biology. 169 (3), 438-449 (2010).

9. Dahmke, I. N. et al. Graphene Liquid Enclosure for Single-Molecule Analysis of Membrane Proteins in Whole Cells Using Electron Microscopy. ACS Nano. 11 (11), 11108-11117 (2017).

10. Maruyama, Y., Ebihara, T., Nishiyama, H., Suga, M., Sato, C. Immuno EM-OM correlative microscopy in solution by atmospheric scanning electron microscopy (ASEM). Journal of Structural Biology. 180 (2), 259-270 (2012). 
11. Kinoshita, T. et al. Immuno-electron microscopy of primary cell cultures from genetically modified animals in liquid by atmospheric scanning electron microscopy. Microscopy and Microanalysis. 20 (2), 469-483 (2014).

12. Hauwiller, M. R., Ondry, J. C., Alivisatos, A. P. Using graphene liquid cell transmission electron microscopy to study in situ nanocrystal etching. Journal of Visualized Experiments. (135), e57665 (2018).

13. Cho, H. et al. The Use of Graphene and Its Derivatives for Liquid-Phase Transmission Electron Microscopy of Radiation-Sensitive Specimens. Nano Letters. 17 (1), 414-420 (2017).

14. Meng, F. et al. Graphene-Based Fibers: A Review. Advanced Materials. 27 (35), 5113-5131 (2015).

15. Sun, P. Z. et al. Limits on gas impermeability of graphene. Nature. 579 (7798), 229-232 (2020).

16. Kato, R. et al. High-precision thickness control of ice layer on CVD grown bilayer graphene for cryo-TEM. Carbon. 160, 107-112 (2020).

17. Keskin, S., de Jonge, N. Reduced Radiation Damage in Transmission Electron Microscopy of Proteins in Graphene Liquid Cells. Nano Letters. 18 (12), 7435-7440 (2018).

18. Giepmans, B. N., Deerinck, T. J., Smarr, B. L., Jones, Y. Z., Ellisman, M. H. Correlated light and electron microscopic imaging of multiple endogenous proteins using Quantum dots. Nature Methods. 2, 743-749 (2005).

19. Ring, E. A., Peckys, D. B., Dukes, M. J., Baudoin, J. P., de Jonge, N. Silicon nitride windows for electron microscopy of whole cells. Journal of Microscopy. 243 (3), 273-283 (2011).
20. Peckys, D. B., Korf, U., de Jonge, N. Local variations of HER2 dimerization in breast cancer cells discovered by correlative fluorescence and liquid electron microscopy. Science Advances. 1 (6), e1500165 (2015).

21. Eigenbrot, C., Ultsch, M., Dubnovitsky, A., Abrahmsen, L., Hard, T. Structural basis for high-affinity HER2 receptor binding by an engineered protein. Proceedings of the National Academy of Sciences of the United States of America. 107 (34), 15039-15044 (2010).

22. Textor, M., de Jonge, N. Strategies for Preparing Graphene Liquid Cells for Transmission Electron Microscopy. Nano Letters. 18, 3313-3321 (2018).

23. Henjes, F. et al. Strong EGFR signaling in cell line models of ERBB2-amplified breast cancer attenuates response towards ERBB2-targeting drugs. Oncogenesis. 1, e16 (2012).

24. Peckys, D. B., de Jonge, N. Studying the Stoichiometry of Epidermal Growth Factor Receptor in Intact Cells using Correlative Microscopy. Journal of Visualized Experiments. (103), e53186 (2015).

25. Pirkmajer, S., Chibalin, A. V. Serum starvation: caveat emptor. American Journal of Physiology. Cell Physiology. 301 (2), C272-279 (2011).

26. Kohl, H., Reimer, L. Transmission electron microscopy: physics of image formation. Springer (2008).

27. Yi, H. et al. Native immunogold labeling of cell surface proteins and viral glycoproteins for cryo-electron microscopy and cryo-electron tomography applications. Journal of Histochemistry and Cytochemistry. 63 (10), 780-792 (2015). 
28. Wolf, S. G., Houben, L., Elbaum, M. Cryo-scanning transmission electron tomography of vitrified cells. Nature Methods. 11 (4), 423-428 (2014).

29. Dukes, M. J., Ramachandra, R., Baudoin, J. P., Jerome, W. G., de Jonge, N. Three-dimensional locations of goldlabeled proteins in a whole mount eukaryotic cell obtained with $3 \mathrm{~nm}$ precision using aberration-corrected scanning transmission electron microscopy. Journal of Structural Biology. 174, 552-562 (2011).

30. Meier, C., Beckmann, A. Freeze fracture: new avenues for the ultrastructural analysis of cells in vitro. Histochemistry and Cell Biology. 149 (1), 3-13 (2018).

31. Peckys, D. B., de Jonge, N. Liquid scanning transmission electron microscopy: imaging protein complexes in their native environment in whole eukaryotic cells. Microscopy and Microanalysis. 20 (2), 346-365 (2014).

32. Egerton, R. F. Control of radiation damage in the TEM. Ultramicroscopy. 127, 100-108 (2013).

33. Wojcik, M., Hauser, M., Li, W., Moon, S., Xu, K. Graphene-enabled electron microscopy and correlated super-resolution microscopy of wet cells. Nature Communications. 6, 7384 (2015).

34. Huebinger, J., Spindler, J., Holl, K. J., Koos, B. Quantification of protein mobility and associated reshuffling of cytoplasm during chemical fixation. Scientific Reports. 8 (1), 17756 (2018).

35. Glauert, A. M., Lewis, P. R. Biological specimen preparation for transmission electron microscopy. Princeton University Press (2014).

36. Koo, K., Dae, K. S., Hahn, Y. K., Yuk, J. M. Live Cell Electron Microscopy Using Graphene Veils. Nano Letters.

20 (6), 4708-4713 (2020).
37. Pawley, J. B. Handbook of biological confocal microscopy. 2 edn, Springer (1995).

38. Chung, I. et al. Spatial control of EGF receptor activation by reversible dimerization on living cells. Nature. 464 (7289), 783-787 (2010). 